\title{
A SANDWICH IN THIN LIE ALGEBRAS
}

\author{
SANDRO MATTAREI (D) \\ Charlotte Scott Research Centre for Algebra, University of Lincoln, Brayford Pool, \\ Lincoln LN6 7TS, UK (smattarei@lincoln.ac.uk)
}

(Received 1 July 2021; first published online 7 January 2022)

\begin{abstract}
A thin Lie algebra is a Lie algebra $L$, graded over the positive integers, with its first homogeneous component $L_{1}$ of dimension two and generating $L$, and such that each non-zero ideal of $L$ lies between consecutive terms of its lower central series. All homogeneous components of a thin Lie algebra have dimension one or two, and the two-dimensional components are called diamonds. Suppose the second diamond of $L$ (that is, the next diamond past $L_{1}$ ) occurs in degree $k$. We prove that if $k>5$, then $[L y y]=0$ for some non-zero element $y$ of $L_{1}$. In characteristic different from two this means $y$ is a sandwich element of $L$. We discuss the relevance of this fact in connection with an important theorem of Premet on sandwich elements in modular Lie algebras.
\end{abstract}

Keywords: Modular Lie algebra; graded Lie algebra; thin Lie algebra; sandwich

2020 Mathematics subject classification: Primary 17B50;

Secondary 17B70; 17B65

\section{Introduction}

A thin Lie algebra is a graded Lie algebra $L=\bigoplus_{i=1}^{\infty} L_{i}$ with $\operatorname{dim}\left(L_{1}\right)=2$ and satisfying the following covering property: for each $i$, each non-zero $z \in L_{i}$ satisfies $\left[z L_{1}\right]=L_{i+1}$. This implies at once that homogeneous components of a thin Lie algebra are at most two-dimensional. Those components of dimension two are called diamonds, hence $L_{1}$ is a diamond, and if there are no other diamonds then $L$ is a graded Lie algebra of maximal class, see $[12,14]$. However, we adopt the convention of explicitly excluding graded Lie algebras of maximal class from the definition of thin Lie algebras. We also require thin Lie algebras to be infinite-dimensional in this paper.

Thus, a thin Lie algebra must have at least one further diamond past $L_{1}$; we let $L_{k}$ be the earliest, and call it the second diamond. It turns out that $k$ can only be one of $3,5, q$, or $2 q-1$, where $q$ is a power of the characteristic $p$ when this is positive. This was proved in [1], but a revised proof is given in [18]. The case of characteristic two, where only 3 and $2 q-1$ can occur, is harder and was settled only in [7]. In particular, only 3 and 5 can occur in characteristic zero. Among the various possibilities for $k$, it is tempting to call the cases where $k$ is 3 or 5 the classical cases, as opposed to the values

(C) The Author(s), 2022. Published by Cambridge University Press on Behalf of The Edinburgh Mathematical Society. This is an Open Access article, distributed under the terms of the Creative Commons Attribution licence (http://creativecommons.org/ licenses/by/4.0/), which permits unrestricted re-use, distribution, and reproduction in any medium, provided the original work is properly cited. 
$q$ and $2 q-1$, which are distinctly modular. The interpretation we provide in $\S 3$ of our main result, Theorem 1, will lend further weight to this informal distinction. Note that in the theory of modular Lie algebras one calls classical those simple Lie algebras which are analogues of the simple Lie algebras of characteristic zero, including the exceptional ones.

Thin Lie algebras with $k$ equal to 3 or 5 , subject to a further restriction $\operatorname{dim}\left(L_{4}\right)=1$ in the former case, and excluding some small positive characteristics, were shown in [13] to belong to (up to) three isomorphism types, associated with $p$-adic Lie groups of the classical types $A_{1}$ and $A_{2}$ (see [17] for matrix realizations of those groups). In contrast, the values $q$ and $2 q-1$ for $k$ occur for two broad classes of thin Lie algebras, of which various families were built from certain non-classical finite-dimensional simple modular Lie algebras, but also to other thin Lie algebras obtained from graded Lie algebras of maximal class through various constructions. General discussions of those two classes of thin Lie algebras may be found in [4] and [11], respectively.

The original motivation for the study of thin Lie algebras came from group theory. A pro- $p$ group $G$ is called thin if $\left|G / \gamma_{2}(G)\right|=p^{2}$, and every open normal subgroup $H$ lies between two consecutive terms of the lower central series of $G$, in the sense that $\gamma_{i}(G) \geq H \geq \gamma_{i+1}(G)$ for some $i$. It is not hard to see that a pro- $p$ group $G$ is thin if and only if the graded Lie ring $L$ associated with its lower central series is a Lie algebra over the field $\mathbb{F}_{p}$ of $p$ elements and satisfies the analogous condition on its graded ideals. In turn, the latter is easily seen to be equivalent to $L$ being a thin Lie algebra over $\mathbb{F}_{p}$. According to [13], the second diamond of the thin Lie algebra associated with a thin pro- $p$ group may only occur in degree 3,5 or $p$. A well-known example of thin pro- $p$ group is the Nottingham group over the field $\mathbb{F}_{p}$, with $p$ odd. Its associated thin Lie algebra has the second diamond in degree $p$ and, more generally, a diamond in each degree congruent to 1 modulo $p-1$, see [8]. Those results prompted investigations of thin Lie algebras as objects of independent study.

Our main result establishes a general fact for thin Lie algebras that was previously shown to hold for various special subclasses in ad hoc manners.

Theorem 1. Let $L$ be a thin Lie algebra with second diamond $L_{k}$, where $k>5$. Then there is a non-zero element $y$ of $L_{1}$ such that $[L y y]=0$.

Here we use the left-normed convention for iterated Lie products, hence $[a b c]$ stands for $[[a b] c]$, and so $[L y y]=0$ is another way of saying $(\operatorname{ad} y)^{2}=0$, where ad denotes the adjoint representation of $L$.

We will prove Theorem 1 in $\S 2$, in an incremental way: we will gradually refine our hypotheses on $L$ to obtain partial results, working our way up to the assumption $k>5$ of Theorem 1, and for each intermediate result there will be remarks showing by examples the necessity of the particular hypotheses assumed or motivating the next step.

According to a definition of Kostrikin, for $p \neq 2$, the conclusion of Theorem 1 says that $y$ is a sandwich element (or simply a sandwich). Kostrikin introduced sandwich elements in the context of the Burnside problem, see [16]. Four decades later, they played an important role in the classification theory of finite-dimensional simple modular Lie algebras in [20], especially for small characteristics (five and seven in this context). One 
remarkable property of sandwich elements, noted by Kostrikin and proved by Premet in [19], is that their presence in a finite-dimensional modular Lie algebra characterizes those simple Lie algebras which are not classical.

Although our thin Lie algebras are infinite-dimensional, a connection with finitedimensional Lie algebras occurs via a loop algebra construction originating from [22], which has then been used in several papers to build thin Lie algebras from finitedimensional Lie algebras, simple or close to simple, with respect to certain cyclic gradings. In $\S 3$, we discuss the relevance of the presence of a sandwich element in this context, and the connection with Premet's result.

For the sake of this Introduction, we outline the example of the thin Lie algebra $L$ associated with the Nottingham group, which has second diamond in degree $p$. A realization of $L$ was given in [8] as a loop algebra of the $p$-dimensional Witt simple algebra $W=W(1 ; 1)$ over $\mathbb{F}_{p}$, which is simply $\operatorname{Der}\left(\mathbb{F}_{p}[X] /\left(X^{p}\right)\right)$. Because $W$ has a grading over the integers, and a $\mathbb{Z}$-graded basis $E_{i}$ for $-1 \leq i \leq p-2$ (with $E_{i}=X^{i+1} d / d X$, say), degree reasons show $E_{p-2}$ is a sandwich element of $W$ if $p>3$ (whence $W$ is not classical if $p>3$ ). In fact, in this case, the sandwich element $y$ of $L$ referred to in Theorem 1 is closely related to the element $E_{p-2}$ of $W$.

\section{A sandwich element in thin Lie algebras}

A sandwich element of a Lie algebra $L$ is a non-zero element $c \in L$ such that $(\operatorname{ad} c)^{2}=0$ and $(\operatorname{ad} c)(\operatorname{ad} z)(\operatorname{ad} c)=0$ for all $z \in L$. The latter requirement (from which the name originates) is superfluous in characteristic different from two, as it follows from the former due to $[u[z c c]]=[u z c c]-2[u c z c]+[u c c z]$ for $u, z \in L$.

In the context of thin Lie algebras, it turns out that a non-zero element $y$ of $C_{L_{1}}\left(L_{2}\right)$ satisfies $[L y y]=0$ in most cases, as our Theorem 1 states, and so $y$ is a sandwich element if the characteristic is not two. This fact was crucial in the theory of graded Lie algebras of maximal class of [14] and [12], making a theory of constituents possible, although the sandwich point of view was not explicitly mentioned there. In particular, [14, Lemma 3.3] can be restated as follows: if $L$ is a graded Lie algebra of maximal class, with the centralizer $C_{L_{1}}\left(L_{2}\right)$ spanned by an element $y$, with $\left[L_{i-1} y\right]=0$ and $\left[L_{i} y\right] \neq 0$ for some $i$, then $\left[L_{i+1} y\right]=0$, whence $\left[L_{i} y y\right]=0$. A remarkable consequence of this fact is $[L y y]=0$, which means $(\operatorname{ad} y)^{2}=0$, and so $y$ is a sandwich element if the characteristic is not two.

Our main goal in this section is showing that such a non-zero element $y$ of $C_{L_{1}}\left(L_{2}\right)$ is frequently a sandwich element also in a thin Lie algebra $L$, under certain assumptions which we introduce and justify along the way. If $L$ has second diamond $L_{3}$, then $C_{L_{1}}\left(L_{2}\right)=0$, hence we must take $\operatorname{dim}\left(L_{3}\right)=1$ as minimal assumption for our discussion, and then $\operatorname{dim}\left(C_{L_{1}}\left(L_{2}\right)\right)=1$. Taking then for $y$ a non-zero element of $C_{L_{1}}\left(L_{2}\right)$ and extending to a basis $x, y$ of $L_{1}$, as we do without further mention in this section, we have $[x y y]=0$.

When $L$ has characteristic two, we limit ourselves to the following simple observation, which (abundantly) covers the case of characteristic two in Theorem 1.

Proposition 2. Let $L$ be a thin Lie algebra of characteristic two, with $\operatorname{dim}\left(L_{3}\right)=1$, and let $y \operatorname{span} C_{L_{1}}\left(L_{2}\right)$. Then $[L y y]=0$. 
Proof. Because $(\operatorname{ad} y)^{2}$ is a derivation of $L$, its kernel is a subalgebra. However, because $[x y y]=0$, both generators $x$ and $y$ of $L$ belong to the kernel, and hence that is the whole of $L$.

We will show that Proposition 2 extends to arbitrary characteristic if we include a further hypothesis $\operatorname{dim}\left(L_{5}\right)=1$. Together with $\operatorname{dim}\left(L_{3}\right)=1$, this amounts to requiring that the second diamond of $L$ occurs later than $L_{5}$, as we assumed in Theorem 1 . A justification for that hypothesis will emerge as we gradually work towards a proof. We start with suitably extending the conclusion $\left[L_{i} y y\right]=0$ of $[14$, Lemma 3.3], which was about graded Lie algebras of maximal class, to certain homogeneous components of any thin Lie algebra $L$ with $\operatorname{dim}\left(L_{3}\right)=1$.

Lemma 3. Let $L$ be a thin Lie algebra with $\operatorname{dim}\left(L_{3}\right)=1$, and let $y \operatorname{span} C_{L_{1}}\left(L_{2}\right)$. Let $L_{i}$ be a homogeneous component with $\left[L_{i} y\right] \neq 0$, and suppose $L_{i-1}$ has a non-zero element $u$ with $[u y]=0$. Then $\left[L_{i} y y\right]=0$. Moreover, $L_{i}$ and $L_{i+2}$ have dimension one.

Proof. Because of the covering property, $L_{i}$ is spanned by $[u x]$, and hence has dimension one. From

$$
0=[u[x y y]]=[u x y y]-2[u y x y]+[u y y x]=[u x y y]
$$

it follows that $\left[L_{i} y y\right]=0$. Because $[u x y] \neq 0$ by hypothesis, the covering property implies that $L_{i+2}$ is spanned by [uxyx], and hence has dimension one.

The following immediate consequence of Lemma 3 resembles the formulation of [14, Lemma 3.3] more closely.

Corollary 4. Let $L$ be a thin Lie algebra with $\operatorname{dim}\left(L_{3}\right)=1$, and let $y \operatorname{span} C_{L_{1}}\left(L_{2}\right)$. If $L_{i-1}$ has a non-zero element centralized by $y$, and $L_{i}$ has none, then $L_{i+1}$ has such an element as well.

Equivalently, in a thin Lie algebra with $\operatorname{dim}\left(L_{3}\right)=1$, at least one of any two consecutive homogeneous components has a non-zero element centralized by $y$. Corollary 4 is often useful as a simple but weaker replacement for Lemma 3. However, we need the full strength of Lemma 3 in order to deduce the following consequence.

Corollary 5. Let $L$ be a thin Lie algebra with $\operatorname{dim}\left(L_{3}\right)=1$, and let $y$ span $C_{L_{1}}\left(L_{2}\right)$. Suppose $\operatorname{dim}\left(L_{j}\right)=2$ for some $j>1$. Then $\operatorname{dim}\left(L_{j-1}\right)=1$ and $\left[L_{j-1} y y\right]=0$. In particular, $L_{j}$ contains a non-zero element centralized by $y$.

Proof. Because of the covering property $L_{j-1}$ cannot contain any non-zero element centralized by $y$. Hence $L_{j-2}$ does contain a non-zero element $u$ with $[u y]=0$. Consequently, $v=[u x]$ spans $L_{j-1}$, and [vyy] $=0$ according to Lemma 3. Because $\operatorname{dim}\left(L_{j}\right)=2$, the element $[v y]$ of $L_{j}$ is non-zero and is centralized by $y$ as desired.

In particular, Corollary 5 implies that two consecutive components in a thin Lie algebra $L$ with $\operatorname{dim}\left(L_{3}\right)=1$ cannot both be diamonds. Thus, the distance between any two consecutive diamonds, by which we mean the difference of their degrees, is at least two. 
An easily proved consequence of this fact is that any thin Lie algebra $L$ with $\operatorname{dim}\left(L_{3}\right)=1$ remains thin under base field extensions. By contrast, this property fails for a thin Lie algebra $L$ with two diamonds occurring as consecutive homogeneous components. This latter fact was never explicitly recorded but was in essence understood since [13], through an explicit calculation showing that the covering property is a quadratic condition in this case, rather than linear. We briefly digress from our main goal to state that result formally, and express its proof in a coordinate-free way.

Proposition 6. Let $L$ be a thin Lie algebra having two consecutive homogeneous components which are both diamonds. Then $L$ ceases to be thin after a suitable quadratic field extension.

Proof. For any graded Lie algebra $L=\bigoplus_{i=1}^{\infty} L_{i}$ we may consider the linear maps $\psi_{j}$ : $L_{j} \rightarrow \operatorname{Hom}_{F}\left(L_{1}, L_{j+1}\right)$ obtained by restriction from the adjoint representation. When $L$ is thin with $L_{j}$ and $L_{j+1}$ both diamonds, the covering property for the homogeneous component $L_{j}$ means that $\psi_{j}\left(L_{j}\right)$ is a two-dimensional subspace of $\operatorname{Hom}_{F}\left(L_{1}, L_{j+1}\right)$ such that every non-zero element is a surjective linear map. Upon composing with a linear bijection $L_{j+1} \rightarrow L_{1}$, whose choice is immaterial, we may view that as a two-dimensional subspace of $\operatorname{Hom}_{F}\left(L_{1}, L_{1}\right)$ where every non-zero element has non-zero determinant. In terms of the associated projective spaces, $P\left(\psi_{j}\left(L_{j}\right)\right)$ is then a one-dimensional projective subspace of the three-dimensional projective space $P\left(\operatorname{Hom}_{F}\left(L_{1}, L_{1}\right)\right)$, disjoint from the non-degenerate quadric given by the zeroes of the determinant map. This condition will be violated under a suitable quadratic extension of the base field of $L$.

The same argument in the proof of Proposition 6 also shows that a thin Lie algebra over a quadratically closed field (a field without extensions of degree two) cannot have two diamonds occurring as consecutive homogeneous components. In fact, the thin Lie algebras studied in $[6,15]$, where all homogeneous components except $L_{2}$ are diamonds, require their base field to have a quadratic field extension.

Returning to our main line of investigation, Corollary 5 is relevant in assigning types to diamonds of a thin Lie algebra. The type of a diamond $L_{j}$ is a scalar in the underlying field, or possibly the symbol $\infty$, which describes certain relations holding in degree $j+1$. When this is done properly (that is, including fake diamonds, see Remark 13 below), the Lie product of a thin Lie algebra (and hence its isomorphism type) is completely determined by specifying all degrees where diamonds occur, and their types. In various notable instances of thin Lie algebras, diamonds occur at regular distances, and their types follow a periodic pattern, such as an arithmetic progression. Attaching types to diamonds needs to be done in different ways according to whether the second diamond occurs in degree $q$ or $2 q-1$, and was originally introduced in [10] and [9], respectively. However, in both cases, necessary conditions for a diamond $L_{j}$ to be assigned a type were $\operatorname{dim}\left(L_{j-1}\right)=1$ and $\left[L_{j-1} y y\right]=0$ (the latter being one of the relations assumed in degree $j+1)$. Corollary 5 now ensures that those conditions hold in any thin Lie algebra with $\operatorname{dim}\left(L_{3}\right)=1$.

Now we use Lemma 3 to prove that if $L$ is thin with $\operatorname{dim}\left(L_{3}\right)=1$, then $\left[L_{i} y y\right]=0$ for all one-dimensional components $L_{i}$. 
Lemma 7. Let $L$ be a thin Lie algebra with $\operatorname{dim}\left(L_{3}\right)=1$, and let $y \operatorname{span} C_{L_{1}}\left(L_{2}\right)$. Suppose $\left[L_{j} y y\right] \neq 0$ for some $j$. Then $\operatorname{dim}\left(L_{j}\right)=2$.

Proof. Note that $j>2$. If $L_{j-1}$ had a non-zero element centralized by $y$, then because $\left[L_{j} y\right] \neq 0$ by hypothesis, Lemma 3 would apply with $i=j$ and yield $\left[L_{j} y y\right]=0$, a contradiction. Therefore, $L_{j-1}$ does not have any non-zero element centralized by $y$. Then because of Corollary 4 each of $L_{j-2}$ and $L_{j}$ has such an element. In particular, because $\left[L_{j} y\right] \neq 0$, we must have $\operatorname{dim}\left(L_{j}\right)=2$.

Thus, the task of proving $[L y y]=0$ in a thin Lie algebra $L$ has now been reduced to showing $\left[L_{j} y y\right]=0$ for the diamonds $L_{j}$. We pause to note that $L$ having any two diamonds at distance two implies $[L y y] \neq 0$. In fact, if $L_{j}$ and $L_{j+2}$ are diamonds, in a thin Lie algebra $L$ with $\operatorname{dim}\left(L_{3}\right)=1$, then the covering property implies $\left[L_{j} y\right] \neq 0$ : the map $\psi_{j}$ of the proof of Proposition 6 is always injective because of the covering property, but it would not be surjective if we had $\left[L_{j} y\right]=0$, and this is impossible here by dimension reasons as $\operatorname{dim}\left(L_{j+1}\right)=1$. Consequently, we have $\left[L_{j} y\right]=L_{j+1}$, and then $\left[L_{j} y y\right]=\left[L_{j+1} y\right] \neq 0$ because $\left[L_{j+1} y\right]$ and $\left[L_{j+1} y\right]$ span the diamond $L_{j+2}$.

The following remark describes one special thin Lie algebra with $\operatorname{dim}\left(L_{3}\right)=1$ having certain diamonds at distance two.

Remark 8. The proof of [13, Theorem 2(a)] implies that in characteristic either zero or larger than five there is a unique (infinite-dimensional) thin Lie algebra $L$ having second diamond $L_{5}$. That result, which predates the study of thin Lie algebras per se, only claims uniqueness for the graded Lie algebra $L$ associated with the lower central series of a infinite thin pro- $p$ group with second diamond in weight 5 , for $p>5$. However, its proof applies to any thin Lie algebra $L$ with second diamond $L_{5}$, for $p>5$ or $p=0$. A construction for that thin Lie algebra $L$ was also given in [13], and that is valid in every characteristic except for characteristic two. The diamonds of $L$ occur in each degree congruent to \pm 1 modulo 6 , and so as noted above one has $\left[L_{i-1} y y\right] \neq 0$ for each $i$ multiple of six. Uniqueness of $L$ fails in characteristic three because $5=2 q-1$ with $q=3$, and in characteristic five because we may have $5=q$ then, and so lots of other thin Lie algebras enter those cases (see Remark 12).

Our next remark describes how in characteristic three one can produce uncountably many thin Lie algebras with $\operatorname{dim}\left(L_{3}\right)=1$ having several diamonds at distance two.

Remark 9. According to [14, Section 9], for each power $q$ of the (positive) characteristic, there are uncountably many infinite-dimensional graded Lie algebras of maximal class $M$ with precisely two distinct two-step centralizers and constituent sequence beginning with $2 q, q, q$. As shown in [9, Section 5], each such $M$ has a maximal subalgebra $L$ which becomes thin under a new grading, and its diamonds start with $L_{1}, L_{2 q-1}, L_{3 q-2}, L_{4 q-3}$. In characteristic three and taking $q=3$ those diamonds are $L_{1}, L_{5}, L_{7}, L_{9}$. Consequently, $\left[L_{5} y y\right] \neq 0$ and $\left[L_{7} y y\right] \neq 0$.

The thin Lie algebras of the above remarks suggest that we might be able to obtain more information on the earliest diamond $L_{j}$ with $\left[L_{j} y y\right] \neq 0$ than on an arbitrary one, hence we will do that in preparation for a proof of Theorem 11. 
Lemma 10. Let $L$ be a thin Lie algebra with $\operatorname{dim}\left(L_{3}\right)=1$, and let $y \operatorname{span} C_{L_{1}}\left(L_{2}\right)$. Suppose $[L y y] \neq 0$, and let $j$ be minimal such $\left[L_{j} y y\right] \neq 0$. Then $L_{j-2}$ and $L_{j-3}$ are onedimensional and centralized by $y$.

Proof. According to Lemma 7, we have $\operatorname{dim}\left(L_{j}\right)=2$, and hence $\operatorname{dim}\left(L_{j-1}\right)=1$ because of Corollary 5 . Note that $j>3$. Let $t$ be any non-zero element of $L_{j-3}$. Then $[t y y]=0$ and $[t x y y]=0$ by minimality of $j$, and hence

$$
0=[t[x y y]]=[t x y y]-2[\operatorname{tyx} y]+[\operatorname{tyy} x]=-2[\operatorname{tyx} y] .
$$

Because of Proposition 2, we are not in characteristic two, and so we conclude [tyxy] $=0$. But then $[t y x]=0$, because $L_{j-1}$ contains no non-zero element centralized by $y$. The covering property now implies $[t y]=0$, otherwise $[t y x]$ and $[t y y]$, which both vanish, would have to span $L_{j-1}$. Because $t$ was an arbitrary non-zero element of $L_{j-3}$, Corollary 5 implies $\operatorname{dim}\left(L_{j-3}\right)=1$. Finally, $L_{j-2}$ has dimension one because it is spanned by $[t x]$. But we know that $L_{j-2}$ contains a non-zero element centralized by $y$, and hence $\left[L_{j-2} y\right]=0$.

We are ready to prove the harder analogue of Proposition 2 for odd characteristics, thus completing a proof of Theorem 1. As our discussion leading to Remark 8 shows, we need an additional assumption to ensure that no diamonds occur at distance two. As it turns out, assuming $\operatorname{dim}\left(L_{5}\right)=1$ will do, which is another way of asking that the second diamond of $L$ occurs past $L_{5}$.

Theorem 11. Let $L$ be a thin Lie algebra of odd characteristic, with $\operatorname{dim}\left(L_{3}\right)=$ $\operatorname{dim}\left(L_{5}\right)=1$, and let $y \operatorname{span} C_{L_{1}}\left(L_{2}\right)$. Then $[$ Lyy $]=0$.

Proof. Besides the relation $[y x y]=0$, which serves to define $y$ up to scalar multiples, in $L$ we also have $[y x x y]=0$ and $[y x x x y]=0$. In fact, the former claim follows from $0=[y x[y x]]=[y x y x]-[y x x y]=-[y x x y]$. As a consequence, $[y x x x]$ spans $L_{4}$. To prove the latter claim, assuming $[y x x x y] \neq 0$ for a contradiction, it will span $L_{5}$ because of our hypothesis $\operatorname{dim}\left(L_{5}\right)=1$. Then because $0=[y x x[x y y]]=[y x x x y y]-2[$ yxxyxy $]+$ $[$ yxxyyx $]=[y x x x y y]$, we find $\left[L_{5} y\right]=0$. Consequently, $[y x x x y x]$ spans $L_{6}$, and we also have $[y x x x x y]=0$. By combining these two facts and computing $0=[y x x x x y]=$ $-[y[y x x x x]]=4[y x x x y x]$, we obtain the desired contradiction.

Now suppose for a contradiction that $[L y y] \neq 0$, and let $j$ be minimal such that $\left[L_{j} y y\right] \neq 0$. We will use the equations $[y x x y]=0$ and $[y x x x y]=0$ to obtain a contradiction. Let $t$ be a non-zero element of $L_{j-3}$. According to Lemma 7 we have $[t y]=0$ and $[t x y]=0$, and $L_{j-1}$ is spanned by $v:=[t x x]$. Furthermore, $\operatorname{dim}\left(L_{j}\right)=2$, and hence $[v y y]=0$ according to Corollary 5 . The calculations

$$
0=[v[x y y]]=[v x y y]-2[v y x y]
$$

and

$$
0=[t[y x x x y]]=[t[y x x x] y]=3[t x x y x y]-[t x x x y y]=-[v x y y]+3[v y x y]
$$

taken together imply $[v x y y]=[v y x y]=0$. Because $[v x]$ and $[v y]$ span $L_{j}$, we obtain $\left[L_{j} y y\right]=0$, which provides the desired contradiction. 
We discuss the extent to which hypothesis $\operatorname{dim}\left(L_{5}\right)=1$ of Theorem 11 is necessary. As we recalled in Remark 8, over a field of characteristic $p>5$ that hypothesis serves to exclude a single exception, given by the unique thin Lie algebra with second diamond $L_{5}$, identified in [13, Theorem 2(a)] and described in Remark 8. However, if we omit the hypothesis $\operatorname{dim}\left(L_{5}\right)=1$ of Theorem 11 in characteristic $p=3$, its conclusion $[$ Lyy $]=0$ is violated by uncountably many thin Lie algebras with second diamond $L_{5}$ and third diamond $L_{7}$, which we described in Remark 9. The following remark focuses on the borderline case $p=5$.

Remark 12. We discuss how far Theorem 11 may be extended to include thin Lie algebras of characteristic five, with second diamond $L_{5}$ and an additional assumption. Countably many thin Lie algebras with second diamond $L_{q}$ were constructed in [4], for any power $q$ of the odd characteristic. In particular, when $q=5$, this gives us a countable family of thin Lie algebras with second diamond $L_{5}$. However, all those thin Lie algebras, which have third diamond $L_{2 q-1}=L_{9}$ (possibly fake, see Remark 13 below), do satisfy the conclusion $[L y y]=0$ of Theorem 11 .

In fact, one can prove that $[L y y]=0$ holds for any thin Lie algebra $L$ of characteristic five satisfying $\operatorname{dim}\left(L_{3}\right)=1, \operatorname{dim}\left(L_{5}\right)=2$, and $\left[L_{7} y\right]=0$. (Here the hypothesis $\left[L_{7} y\right]=0$ implies that neither $L_{7}$ nor $L_{8}$ is a diamond, and hence the third diamond of $L$ does not occur earlier than $L_{9}$.) A proof of this fact follows the general inductive strategy employed in [2], but is not a formal consequence of results stated in [2], where some generality was sacrificed in favour of a simpler exposition. In fact, part of that simplification in [2] relies on using our Theorem 11.

Remark 13. We mentioned fake diamonds a couple of times in this section, in marginal comments to our main discussion. When assigning types to diamonds of a thin Lie algebra $L$ with second diamond $L_{q}$ (see [10]) or $L_{2 q-1}$ (see [9]), it is convenient for descriptive purposes to allow certain one-dimensional homogeneous components to be called fake diamonds (of type 0 or 1 in the former case, and of type 0 in the latter). In some situations, it may even make sense to allow the second diamond of $L$ to be fake; this may, in fact, be viewed as the source of serious complications in characteristic two, see [4, Remarks 3.6 and 5.3], and [7] for their resolution. For the avoidance of doubt, each reference to unqualified diamonds of a thin Lie algebra in the present paper is to genuine diamonds, that is, two-dimensional homogeneous components.

\section{Thin loop algebras and the sandwich element}

We conclude this paper with a discussion of the significance for thin Lie algebras of an important property of sandwich elements. Classical simple Lie algebras do not have sandwich elements, see [21, p. 124]. By contrast, confirming a conjecture of Kostrikin, Premet proved in [19] that every finite-dimensional simple Lie algebra, over an algebraically closed field of characteristic $p>5$, which is not classical, must have sandwich elements (that is, have strong degeneration).

The connection of this characterization of classical Lie algebras with (infinitedimensional) thin Lie algebras comes from the fact that several of the latter have been constructed as loop algebras of certain finite-dimensional simple Lie algebras, or close to 
simple. In the simplest setting, one starts from a finite-dimensional simple Lie algebra $S$ over a field $F$, with a cyclic grading $S=\bigoplus_{k \in \mathbb{Z} / N \mathbb{Z}} S_{k}$, and considers the Lie algebra $S \otimes F[t]$ over $F$, where $t$ is an indeterminate. Its subalgebra $L=\bigoplus_{k>0} S_{\bar{k}} \otimes t^{k}$, where $\bar{k}=k+N \mathbb{Z}$, is naturally graded over the positive integers, and is called a loop algebra in this context. In certain cases, one needs a slightly more general construction involving also a derivation of $S$ (such as that of [4, Definition 2.1]), but that is inconsequential for our present observation.

It was proved in [13] that a thin Lie algebra $L$ (of characteristic not 2 or 3 ) having second diamond $L_{3}$ and $\operatorname{dim}\left(L_{4}\right)=1$ belongs to one of (up to) two isomorphism types, and a thin Lie algebra $L$ (of characteristic not 2, 3 or 5 ) with second diamond $L_{5}$ is unique up to isomorphism (see also Remark 8). Each of those thin Lie algebras can be realized as a loop algebra of a classical simple Lie algebra of type $A_{1}$ or $A_{2}$.

Now, the fact that those thin Lie algebras of [13] are loop algebras of classical simple Lie algebras implies that there cannot be any non-zero element $y \in L_{\overline{1}}$ with $(\operatorname{ad} y)^{2}=0$, because such $y$ would have the form $y=c \otimes t$ for some sandwich element $c$ of $S$, which cannot exist. For the same reason, if a thin Lie algebra $L$ (say of characteristic $p>5$ ) with second diamond past $L_{5}$ is a loop algebra of a simple Lie algebra $S$, then $S$ cannot be classical, because $L$ has a sandwich element $y$ according to Theorem 11, and hence so does $S$.

In fact, all constructions of thin Lie algebras with second diamond $q$ or $2 q-1$ as loop algebras which were given in several papers $[3-5,8,11]$ start from non-classical simple Lie algebras of various types. The occurrence of non-classical simple Lie algebras in those constructions was hardly surprising in itself, because the expected structure of such thin Lie algebras $L$ given by corresponding uniqueness results showed that the dimension of $S$ ought to be a power of $p$ or one or two less in the various cases. However, the sandwich element $y$ did provide guidance in identifying the appropriate cyclic grading of $S$ employed in those constructions.

\section{Competing interests}

The author declares none.

\section{References}

1. M. Avitabile and G. Jurman, Diamonds in thin Lie algebras, Boll. Unione Mat. Ital. Sez. B Artic. Ric. Mat. (8) 4(3) (2001), 597-608.

2. M. Avitabile and S. Mattarei, Diamond distances in Nottingham algebras. arXiv:2011. 05491, to appear in J. Algebra Appl.

3. M. Avitabile and S. Mattarei, Thin Lie algebras with diamonds of finite and infinite type, J. Algebra 293(1) (2005), 34-64.

4. M. Avitabile and S. Mattarei, Thin loop algebras of Albert-Zassenhaus algebras, J. Algebra 315(2) (2007), 824-851.

5. M. Avitabile and S. Mattarei, Nottingham Lie algebras with diamonds of finite and infinite type, J. Lie Theory 24(2) (2014), 457-473.

6. M. Avitabile, A. Caranti, N. Gavioli, V. Monti, M. F. Newman and E. A. O'BriEn, Thin subalgebras of Lie algebras of maximal class. arXiv:2101.11982, to appear in Israel J. Math. 
7. M. Avitabile, G. Jurman and S. Mattarei, The structure of thin Lie algebras with characteristic two, Inter. J. Algebra Comput. 20(6) (2010), 731-768.

8. A. Caranti, Presenting the graded Lie algebra associated to the Nottingham group, J. Algebra 198(1) (1997), 266-289.

9. A. Caranti and S. Mattarei, Some thin Lie algebras related to Albert-Frank algebras and algebras of maximal class, J. Austral. Math. Soc. Ser. A 67(2) (1999), 157-184.

10. A. Caranti and S. Mattarei, Nottingham Lie algebras with diamonds of finite type, Inter. J. Algebra Comput. 14(1) (2004), 35-67.

11. A. Caranti and S. Mattarei, Gradings of non-graded Hamiltonian Lie algebras, J. Aust. Math. Soc. 79(3) (2005), 399-440.

12. A. Caranti and M. F. Newman, Graded Lie algebras of maximal class. II, J. Algebra 229(2) (2000), 750-784.

13. A. Caranti, S. Mattarei, M. F. Newman and C. M. Scoppola, Thin groups of prime-power order and thin Lie algebras, Quart. J. Math. Oxford Ser. (2) 47(187) (1996), 279-296.

14. A. Caranti, S. Mattarei and M. F. Newman, Graded Lie algebras of maximal class, Trans. Amer. Math. Soc. 349(10) (1997), 4021-4051.

15. N. Gavioli, V. Monti and D. S. Young, Metabelian thin Lie algebras, J. Algebra 241(1) (2001), 102-117.

16. A. I. Kostrikin, Around Burnside, volume 20 of Ergebnisse der Mathematik und ihrer Grenzgebiete (3) [Results in Mathematics and Related Areas (3)].( Springer-Verlag, Berlin, 1990). Translated from the Russian and with a preface by James Wiegold.

17. S. Mattarei, Some thin pro-p-groups, J. Algebra 220(1) (1999), 56-72.

18. S. Mattarei, Constituents of graded Lie algebras of maximal class and chains of thin Lie algebras, Comm. Algebra (2021). doi:10.1080/00927872.2021.1967368.

19. A. A. Premet, Lie algebras without strong degeneration, Mat. Sb. (N.S.) 129(171(1)) (1986), 140-153. [In Russian]; English translation, Math. USSR-Sb. 57 (1987), 151-164.

20. A. Premet and H. Strade, Simple Lie algebras of small characteristic. I. Sandwich elements, J. Algebra 189(2) (1997), 419-480.

21. G. B. Seligman, Modular Lie algebras. Ergebnisse der Mathematik und ihrer Grenzgebiete, Band 40. (Springer-Verlag New York, Inc., New York, 1967).

22. A. Shalev, Simple Lie algebras and Lie algebras of maximal class, Arch. Math. (Basel) 63(4) (1994), 297-301. 